\title{
Assessing the in vivo impact of a gel sanitizer on the epidermal "barrier" dynamics
}

\section{Avaliação do impacto in vivo de um gel desinfetante na dinâmica da "barreira" epidérmica}

\author{
Henrique Silva ${ }^{1,2}$, Sara Aguiar Silva ${ }^{2}$, Hugo Ferreira ${ }^{3}$ and L. Monteiro Rodrigues ${ }^{1,2}$ \\ ${ }^{1}$ CBIOS - Universidade Lusófona's Research Center for Biosciences and Health Technologies (UDE), Campo Grande, 376, \\ 1749-024, Lisboa, Portugal \\ ${ }^{2}$ Pharmacol. Sc Depart - Universidade de Lisboa, School of Pharmacy, Lisboa, Portugal \\ ${ }^{3}$ Institute of Biophysics and Biomedical Engineering, Faculty of Sciences of the University of Lisbon \\ E-mail: henrique.silva@ulusofona.pt
}

*This project is supported by the grant PADDIC 2013-2014, from ALIES-COFAC and part of the PhD Program in Health Sciences from U Alcalá and U Lusófona

*Este projeto é apoiado pela bolsa PADDIC 2013-2014, da ALIES-COFAC e faz parte do Programa de Doutoramento em Ciências da Saúde da parceria U Alcalá - U Lusófona

\begin{abstract}
Disease prevention and control depend on hand washing, in particular during epidemic surges (e.g. flu). The use of alcohol-based hand sanitizers is strongly recommended due to its high germicide effectiveness. However, its impact on skin physiology, especially on the barrier function, has not been determined, although most of the formulations include different humectants. This study evaluates the impact of a commercially available formulation on in vivo epidermal barrier dynamics. 13 young adult subjects (21.6 \pm 2.6 years old) were selected after informed consent. Subjects were asked to apply a commercially available alcohol gel sanitizer to one hand for 15 days. TEWL absolute values were measured on days 1,8 and 15 and a plastic occlusion stress test (POST) applied. Evaporation half-life $\left(\mathrm{t}_{1 / 2 \text { evap }}\right)$ and dynamic water mass $(D W M)$ values increased significantly on day 15 on the treated hand compared to the control hand suggesting that, in the present experimental conditions, the regular use of an alcohol-based gel sanitizer does changes the epidermal barrier. This study also confirms the usefulness of this kinetic model to detect subtle changes on in vivo TEWL dynamics.
\end{abstract}

Keywords: hand sanitizer; epidermal barrier; TEWL; dynamical assessment; POST

\section{Resumo}

O controlo e prevenção de doenças têm, na lavagem das mãos, um instrumento principal, em particular durante surtos epidémicos (ex: gripe). Recomenda-se a utilização de desinfetantes para mãos à base de álcool pela sua elevada eficácia germicida. Mas, o seu impacto sobre a fisiologia da pele, e a função de barreira em especial, não está avaliado, embora a maioria das formulações inclua diferentes humectantes. Este estudo avalia o impacto deste tipo de formulação sobre a dinâmica da barreira epidérmica in vivo. 13 indivíduos saudaveis (21.6 \pm 2.6 anos) foram selecionados após consentimento informado. Os participantes foram instruídos a lavar as mãos durante 15 dias com um desinfectante de maõs disponível no mercado. Os valores da PTEA foram medidos nos dias 1, 8 e 15, aplicando-se ainda um teste POST. Os parâmetros tempo de semi-vida de evaporação $\left(\mathrm{t}_{1 / 2}\right.$ evap $)$ e dinâmica da massa de água $(D W M)$ aumentaram significativamente no dia $15 \mathrm{em}$ especial na mão tratada, sugerindo que, nas presentes condições experimentais, o uso regular deste gel altera a barreira epidérmica. Este estudo também confirma a utilidade do modelo cinético para detectar mudanças mesmo que discretas na dinâmica in vivo da PTEA.

Palavras-chave: desinfetante de mãos; barreira epidérmica; PTEA; avaliação dinâmica; POST 


\section{Introduction}

Hand washing has been referred as critical to promote disease control and prevention ${ }^{[1]}$, in particular during epidemic surges (e.g. flu) ${ }^{[2]}$ and has become a mandatory practice in healthcare settings ${ }^{[3,4]}$. Alcohol-based hand sanitizers - mostly in gel form, are easier to use and require shorter drying times compared with standard detergent-based products ${ }^{[5]}$. Alcohol-based products with $60-95 \%$ alcohol content have been found to be more effective in killing germs than those with lower alcohol concentration or non-alcohol-based hand sanitizers ${ }^{[4]}$. However, several complaints, such as dryness, the most frequent, have been presented as cause of reduced compliance distracting from the regular handcleaning procedure ${ }^{[6,7]}$. For this reason, a new generation of hand sanitizers were recently introduced in the market, exploring different advantages from cosmetic industry experience and knowledge of skin physiology in order to overpass these concerns and to promote its regular (more frequent) use, in particular in specific environments (hospitals, health centers), and to contribute to a more effective disease control and prevention.

Transepidermal Water Loss (TEWL) is the most commonly assessed variable to quantify the epidermal 'barrier' function in vivo ${ }^{[8]}$. In order to improve the precision of TEWL measurements, other dynamical approaches have been suggested. The moisture accumulation test (MAT) ${ }^{[9]}$, the sorption/desorption test ${ }^{[10]}$, and the plastic occlusion stress test (POST) ${ }^{[11]}$ are relevant examples serving as the conceptual basis for the compartmental model here applied, allowing a more accurate quantitative description of the cutaneous water mass balance over time ${ }^{[12-14]}$.

This study evaluates the impact of the use of an alcoholbased gel sanitizer on the epidermal 'barrier' function, using a kinetic approach of TEWL desorption curves following a POST test.

\section{Materials and Methods}

13 healthy subjects (mean $21.6 \pm 2.6$ years old), both genders, were enrolled after informed written consent. The study was developed in accordance with the Declaration of Helsinki and its subsequent amendments ${ }^{[15]}$. All volunteers applied $4 \mathrm{~mL}$ of an $80 \%$ (v/v) commercially available alcohol-containing gel ( $\mathrm{Gel}$ desinfectante de mãos, Holon Produtos) on the dorsum of one randomly chosen hand, twice-daily for 15 consecutive days. The gel composition is listed in Table 1. After

\section{Introdução}

A lavagem das mãos tem sido apontada como fundamental para promover o controlo e prevenção de doenças ${ }^{[1]}$, em particular durante surtos epidémicos (ex: gripe) ${ }^{[2]}$ e tornou-se uma prática obrigatória em serviços de saúde ${ }^{[3,4]}$. Os desinfetantes para as mãos à base de álcool, na sua maioria gelificados, são mais fáceis de utilizar e requerem menor tempo de secagem em comparação com produtos padrão à base de detergentes ${ }^{[4]}$. Os produtos com $60-95 \%$ de teor de álcool têm sido considerados germicidas mais eficazes que aqueles com menor concentração de álcool ou higienizadores sem base alcoólica ${ }^{[4]}$. No entanto, várias queixas como a secura, a mais frequente, têm sido referidas como causa de redução da adesão e não motivação para a lavagem regular das mãos ${ }^{[6,7]}$. Por esta razão, uma nova geração de desinfetantes para as mãos foi recentemente introduzida no mercado, explorando diferentes vantagens do conhecimento e experiência da indústria de cosméticos e da fisiologia da pele, a fim de ultrapassar essas preocupações e promover o seu uso mais frequente, em particular em ambientes específicos (hospitais, centros de saúde), de forma a contribuir para um controlo e prevenção da doença mais eficazes.

A perda transepidérmica de água (PTEA) é a variável mais utilizada para quantificar a função de 'barreira' epidérmica in vivo ${ }^{[8]}$. A fim de melhorar a precisão das medições da PTEA, outras abordagens dinâmicas têm sido sugeridas. Os testes de acumulação de humidade (MAT) ${ }^{[9]}$, de sorção/dessorção ${ }^{[10]}$ e de oclusão plástica (POST) ${ }^{[11]}$ são exemplos relevantes que servem como base conceptual para o modelo aqui aplicado, permitindo uma descrição quantitativa mais precisa do balanço de massa de água cutânea ao longo do tempo ${ }^{[12-14]}$.

O presente estudo avalia o impacto da utilização de um gel desinfetante de base alcoólica sobre a função de 'barreira' epidérmica, usando uma abordagem de modelação cinética das curvas de dessorção da PTEA após um POST.

\section{Materiais e métodos}

13 indivíduos saudáveis, (média de $21.6 \pm 2.6$ anos), de ambos os géneros, participaram neste estudo após consentimento informado por escrito. $\mathrm{O}$ estudo foi desenvolvido em conformidade com a Declaração de Helsínquia e suas emendas subsequentes ${ }^{[15]}$. Os voluntários aplicaram $4 \mathrm{~mL}$ de um gel desinfetante contendo álcool a $80 \%(\mathrm{v} / \mathrm{v})$ disponível no mercado (Gel desinfectante de mãos, Holon Produtos) no dorso de uma mão escolhida aleatoriamente, duas vezes por dia durante 15 dias 
application the gel was left to dry in the treated hand, while the contralateral hand served as control. Subjects were instructed to refrain from using any hand care products during the study.

TEWL, expressed in $\left(\mathrm{g} / \mathrm{m}^{2}\right) / \mathrm{h}$, was measured in the hand dorsal face by evaporimetry (Tewameter TM300, Courage \& Khazaka, Cologne, Germany). Measurements took place in days $1\left(t_{0}\right), 8\left(t_{1}\right)$ and $15\left(t_{2}\right)$ in an area corresponding to the middle of the second and third fingers. Additionally, on days 1 and 15 the region between the first and second fingers on the dorsum of each hand was occluded for 24 hour by an occlusive patch. The patch consisted of four layers, a Parafilm ${ }^{\mathrm{TM}}$ (Bemis Flexible Packaging, Neenah, WI, USA) layer in contact with the skin, cling film, a second Parafilm ${ }^{\mathrm{TM}}$ layer and finally a Trumed plaster (Trumed Technologies Inc., Burnsville, USA) on the top (Figure 1). consecutivos. A composição do gel está indicada na Tabela 1. Após a aplicação, o gel foi deixado secar na mão tratada, enquanto que a mão contralateral serviu de controlo. Os participantes foram instruídos a absterem-se de aplicar qualquer produto tópico na mão durante o estudo.

A PTEA, expressa em $\left(\mathrm{g} / \mathrm{m}^{2}\right) / \mathrm{h}$, foi medida no dorso da mão por evaporimetria (Tewameter TM300, Courage \& Khazaka, Colónia, Alemanha). As medidas foram realizadas no dia $1\left(\mathrm{t}_{0}\right), 8\left(\mathrm{t}_{1}\right)$ e $15\left(\mathrm{t}_{2}\right)$ numa área situada entre o segundo e terceiro dedos. Adicionalmente, nos dias 1 e 15 a região dorsal entre o primeiro e segundo dedos de cada mão foi ocludida durante 24 horas com um penso oclusivo. O penso oclusivo foi construído com quatro camadas - uma folha de Parafilm ${ }^{\mathrm{TM}}$ (Bemis Flexible Packaging, Neenah, WI, EUA) em contato com a pele, uma película aderente, uma outra camada de Parafilm ${ }^{\mathrm{TM}}$ e, finalmente, um penso Trumed (Trumed Technologies Inc., Burnsville, EUA) no topo (Figura 1).

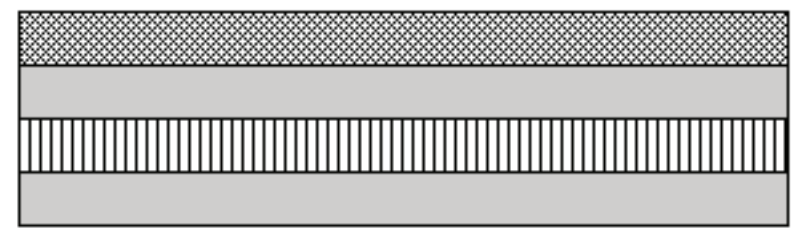

Trumed

Parafilm ${ }^{\text {IM }}$

Cling film

Parafilm ${ }^{1 \mathrm{M}}$

Figure 1/ Figura 1. Schematic illustration of the occlusive patch used

\section{Ilustração esquemática do penso oclusivo utilizado}

The occlusive patch causes a significant occlusion to the skin, causing it to retain water. After patch removal, $T E W L$ initially increases and then progressively decays towards its normal basal level. TEWL was continuously measured for 30 minutes, at a frequency of $0.1 \mathrm{~Hz}$ (one measurement every ten seconds) and modelled by a bicompartment model. This model, first proposed in 1999 ${ }^{[12]}$, considers the barrier and inner layers of the skin as two different water compartments. The first compartment is the skin barrier with low water content, while the second represents the innermost layers of the skin with higher water levels. The TEWL decay curve can be described by the following equation (Eq.1):

$$
T E W L=B+I\left(e^{-K_{\text {evap }} \times t}-e^{-K_{\text {hydr }} \times t}\right),(\text { Eq. } 1)
$$

where $B$ represents the baseline effect, the finite amount of water permanently lost through skin; $I$ the common multiplicative exponential; $K_{\text {evap }}$, the evaporation constant in assessing barrier function; and $K_{\text {hydr }}$ hydration rate constant, related to the distribution of water by two compartments.
A oclusão leva à retenção de água e, após a sua remoção a PTEA aumenta inicialmente e diminui progressivamente até ao seu nível basal normal. A PTEA foi medida continuamente durante 30 minutos a uma frequência de $0,1 \mathrm{~Hz}$ (uma medição a cada dez segundos) e modelada por um modelo bi-compartimental, proposto pela primeira vez em $1999^{[12]}$, que considera a barreira e as camadas internas da pele como dois compartimentos hídricos diferentes. O primeiro compartimento representa a barreira da pele com baixo teor de água, enquanto o segundo representa as camadas mais internas da pele com níveis mais elevados de água. A curva de decaímento de $P T E A$ pode ser descrita pela seguinte equação 1 (Eq.1):

$$
\text { PTEA = B + I ( }\left(\mathrm{e}^{-\mathrm{K}_{\text {evap }} \times \mathrm{t}}-\mathrm{e}^{-\mathrm{K}_{\text {hidr }} \times \mathrm{t}}\right), \quad \text { (Eq.1) }
$$

onde $B$ representa o efeito basal, a quantidade finita de água permanentemente perdida através da pele; $I$ o exponencial multiplicativo comum; $K_{\text {evap }}$, a constante de evaporação na avaliação da função de barreira, e $K_{\text {hydr }}$ a taxa de hidratação, relacionada com a distribuição de água pelos dois compartimentos. 
To facilitate data interpretation, $K_{\text {evap }}$ can be transformed into $t_{1 / 2 \text { evap }}$ (half-life of evaporation) as represented in equation 2 (Eq.2):

$$
\mathrm{t}_{1 / 2 \text { evap }}=\frac{\ln 2}{\mathrm{~K}_{\text {evap }}}
$$

where $t_{1 / 2 \text { evap }}$ is the time required by the body to reduce its water loss by half.

Another parameter used in data analysis is the dynamic water mass $(D W M)$, which represents the relevant weight of water involved in the process of desorption. This parameter is obtained by calculating the area under the curve (AUC) of $t_{\max }$ until the end of the experiment (Eq.3):

$$
D W M=\int_{t_{\max }}^{t} B+I\left(e^{-K_{\text {evap }} \times t}-e^{-K_{\text {hydr }} \times t}\right), \quad(\text { Eq. 3) }
$$

The mathematical model was adjusted to the data points using a Levenberg-Marquadt algorithm and the software MatLab (MATLAB and Statistics Toolbox Release 2011, The MathWorks, Inc., Natick, Massachusetts, USA).

The evolution of the absolute TEWL values and of curve-derived kinetic parameters were compared for both hands and between the control and treated hands by the Wilcoxon signed-rank test $(p<0.05)$.

\section{Results and Discussion}

Table 2 includes TEWL data obtained in $t_{0}, t_{1}$ and $t_{2}$ and the respective statistical comparison between hands, while Table 3 includes the differences between variables in the same measurement days.

As shown, TEWL increased progressively during the study but significant differences could only be found on day 15 , especially regarding the treated hand respective to the control. These results indicate that, in the present experimental conditions, the hand sanitizer gel does affect the epidermal barrier function, no matter the humectants included in the formulation (Table 1). This 'barrier' alteration by organic solvents has been demonstrated and approached in different perspectives. Ethanol seems to induce the fluidification of the intercellular lipid matrix ${ }^{[16]}$ by extracting lipid components ${ }^{[17]}$ and also modifying stratum corneum keratin conformation ${ }^{[17]}$, clearly modifying epidermal barrier efficacy and increasing TEWL ${ }^{[14]}$.
Para facilitar a interpretação dos dados, $K_{\text {evap }}$ pode ser transformada em $t_{1 / 2 \text { evap }}$ (tempo de semi-vida de evaporação), tal como representado na equação 2 (Eq.2):

$$
\mathrm{t}_{1 / 2 \text { evap }}=\frac{\ln 2}{\mathrm{~K}_{\text {evap }}}
$$

onde $t_{1 / 2 \text { evap }}$ é o tempo necessário para reduzir a perda de água para metade.

Outro parâmetro utilizado na análise dos dados é a dinâmica da massa de água envolvida em todo o processo $(D W M)$, que representa o peso relevante de água envolvido no processo de dessorção. Este parâmetro é obtido através do cálculo da área sob a curva $(A S C)$ de $t_{\text {máx }}$ até ao fim do registo (Eq.3):

$D M A=\int_{t_{\text {max }}}^{t} B+I\left(e^{-K_{\text {evap }} \times t}-e^{-K_{\text {hydr }} \times t}\right),($ Eq. 3)

O modelo matemático foi ajustado para os pontos experimentais usando um algoritmo de Levenberg-Marquadt e o software MatLab (MATLAB e Estatística Toolbox Versão 2011, The MathWorks, Inc., Natick, Massachusetts, EUA).

A evolução dos valores absolutos de PTEA e dos parâmetros cinéticos foi comparada entre as duas mãos e entre a mão controlo e a mão tratada, com o teste não paramétrico de Wilcoxon para amostras emparelhadas $(p<0.05)$.

\section{Resultados e discussão}

A Tabela 2 inclui dados de PTEA obtidos em $t_{0}, t_{1}$ e $t_{2} \mathrm{e}$ a respectiva comparação estatística entre as mãos, e na Tabela 3 apresentam-se as diferenças entre as variáveis nos mesmos dias de medição.

Conforme se observa, a PTEA aumentou progressivamente ao longo do estudo, mas diferenças significativas só foram encontradas no dia 15 , especialmente na mão tratada relativamente à controlo. Isto significa que, nas presentes condições experimentais, o álcool gel afeta a função de 'barreira' epidérmica, independentemente da presença dos humectantes incluídos na formulação (Tabela 1). A alteração provocada por solventes orgânicos sobre a barreira epidérmica, tem sido demonstrada e abordada sob diferentes perspectivas. O etanol parece induzir a fluidificação da matriz lipídica intercelular ${ }^{[16]}$ por extração dos componentes lipídicos ${ }^{[17]}$, e também ao modificar a conformação da queratina do estrato córneo ${ }^{[17]}$, 
In order to better understand the involved water dynamics, a previously developed measurement strategy involving a controlled stress test to modify the epidermal water content (POST) was applied. After a 24 hour occlusion the TEWL decay curves profiles were obtained and analyzed by an algorithm ${ }^{[12]}$ that transforms these phenomena into kinetic parameters $\left(t_{1 / 2 \text { evap }}\right.$ and $\left.D W M\right)$. This model has been tested in multiple circumstances and has been proven to be particularly sensitive and rigorous ${ }^{[12-14]}$. Typical TEWL decay curves are shown in Figure 2.

As seen in Table 4, clear consistent differences were found for the kinetic parameters determined between days 1 and 15 in both hands. In fact, $t_{1 / 2 \text { evap }}$ increased significantly on both hands between days 1 and 15, although more pronounced in the treated hand. The $D W M$ was also much higher in the treated hand compared with non-treated, but significant differences could not be found. modificando claramente a eficácia da barreira epidérmica e provocando um aumento da $P T E A^{[14]}$.

A fim de entender melhor a dinâmica da água envolvida, aplicámos uma estratégia de medição desenvolvida anteriormente pela aplicação de um teste de provocação, o POST para modificar o teor de água da epiderme. Depois de uma oclusão de $24 \mathrm{~h}$, os perfis das curvas de decaímento da PTEA são analisados pelo algoritmo do modelo que transforma estes fenómenos em parâmetros cinéticos $\left(t_{1 / 2 \text { evap }} \text { e } D W M\right)^{[12]}$. Este modelo foi testado em diversas circunstâncias e provou ser particularmente sensível e rigoroso ${ }^{[12-14]}$. Curvas típicas de decaímento da PTEA são mostradas na Figura 2 pode ser visto na Tabela 4 existem diferenças consistentes para os parâmetros cinéticos determinados entre os dias 1 e $15 . t_{1 / 2 \text { evap }}$ aumentou significativamente em ambas as mãos entre os dias 1 e 15 , revelando maiores taxas de eliminação de água inicial e do tempo de semi-vida de evaporação. A $D W M$ também aumentou em ambas as mãos, especialmente na mão tratada, mas nas presentes condições experimentais, não encontrámos diferenças significativas.
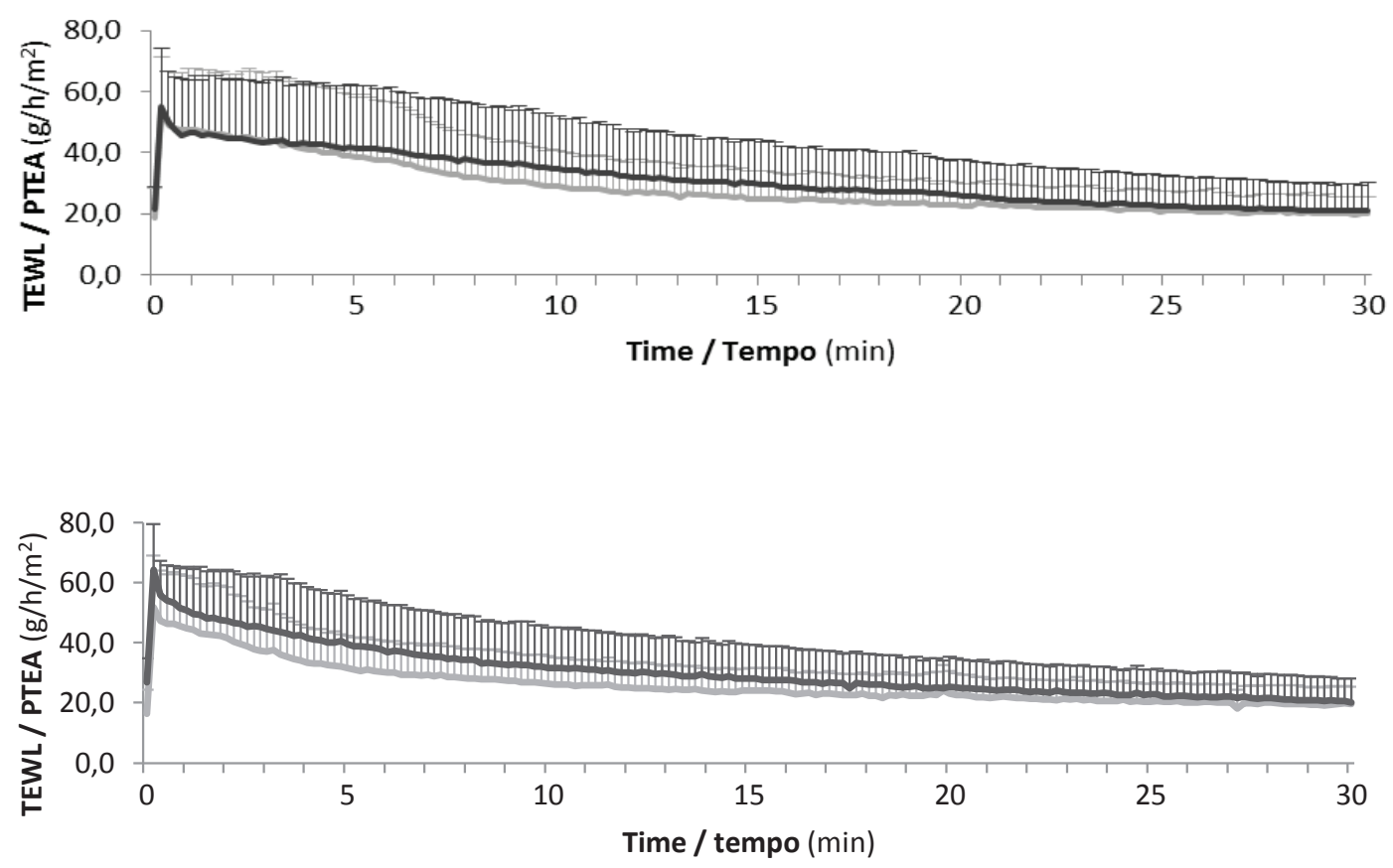

Figure 2/ Figura 2. Continuous TEWL recording during 30 minutes after occlusive patch removal (vertical bars represent standard deviation; light grey - day 1; dark grey day 15) for the control (upper) and the treated (down) hands.

Registo contínuo de PTEA durante 30 minutos após remoção do penso oclusivo (barras verticais representam o desvio-padrão; cinzento claro - dia 1; cinzento escuro - dia 15) para as mãos controlo (em cima) e tratada (em baixo). 
Table 1/ Tabela 1. Hand sanitizer gel composition Composição do gel desinfetante

\begin{tabular}{|c|c|}
\hline $80 \%(\mathrm{v} / \mathrm{v})$ ethanol / Etanol $80 \%(\mathrm{v} / \mathrm{v})$ & Triethanolamine / Trietanolamina \\
\hline Water / Água & Parfum / Perfume \\
\hline Propylene glycol / Propilenoglicol & Limonene / Limoneno \\
\hline Glycerin / Glicerina & $\begin{array}{l}\text { Alfa-isomethyl ionone / Ionona alfa- } \\
\text { isometílica }\end{array}$ \\
\hline $\begin{array}{l}\text { acrylate/acrylic copolymer / Copolímero de } \\
\text { ácido acrílico/acrilato }\end{array}$ & Benzyl salicylate / Salicilato de benzilo \\
\hline Triclosan & $\begin{array}{l}\text { Butylphenyl methylpropional / Metilpropional } \\
\text { de butilfnilo }\end{array}$ \\
\hline Aloe Barbadensis "leaf juice" & Citronellol / Citronelol \\
\hline Carbomer / Carbómero & Hexyl cinnamal / Cinamal de hexilo \\
\hline
\end{tabular}

Table 2/ Tabela 2. Results (mean \pm standard deviation) obtained for Transepidermal Water Loss (TEWL) for the three measurement moments $\left(\mathrm{t}_{0}-\right.$ day $1 ; \mathrm{t}_{1}-$ day $8 ; \mathrm{t}_{2}-$ day $15)$. Comparative statistics is also shown $(* \mathrm{p}<0.05)$

Resultados (média \pm desvio-padrão) obtidos para a Perda Transepidérmica de Água (PTEA) nos três momentos de medição $\left(\mathrm{t}_{0}-\right.$ dia $1 ; \mathrm{t}_{1}-$ dia $8 ; \mathrm{t}_{2}-$ dia 15$)$. A estatística comparativa é também mostrada $(* \mathrm{p}<0.05)$

\begin{tabular}{|c|c|c|c|c|c|c|c|c|c|}
\hline \multirow{3}{*}{\multicolumn{2}{|c|}{$\begin{array}{l}\text { Variable / } \\
\text { Variável }\end{array}$}} & \multirow{3}{*}{$\begin{array}{l}\text { Control / } \\
\text { Controlo }\end{array}$} & \multirow{3}{*}{$\begin{array}{c}\text { Treated / } \\
\text { Tratada }\end{array}$} & \multicolumn{6}{|c|}{ p value / valor p } \\
\hline & & & & \multicolumn{3}{|c|}{ Control / Controlo } & \multicolumn{3}{|c|}{ Treated / Tratada } \\
\hline & & & & $\mathbf{t}_{\mathbf{0}} \mathbf{v s} \mathbf{t}_{1}$ & $t_{0}$ vs $t_{2}$ & $t_{1}$ vs $t_{2}$ & $\mathbf{t}_{\mathbf{0}} \mathbf{v s \mathbf { t } _ { 1 }}$ & $t_{0}$ vs $t_{2}$ & $\mathbf{t}_{1}$ vs $\mathbf{t}_{2}$ \\
\hline $\begin{array}{l}\text { TEWL / } \\
\text { PTEA } \\
\left(\mathrm{g} / \mathrm{h} / \mathrm{m}^{2}\right)\end{array}$ & $\begin{array}{l}\mathbf{t}_{0} \\
\mathbf{t}_{1} \\
\mathbf{t}_{2}\end{array}$ & $\begin{array}{l}11.9 \pm 3.3 \\
12.5 \pm 4.3 \\
12.1 \pm 3.0\end{array}$ & $\begin{array}{l}11.7 \pm 2.3 \\
12.9 \pm 3.9 \\
13.8 \pm 3.0\end{array}$ & 0.507 & 0.875 & 0.695 & 0.133 & $0.012 *$ & 0.108 \\
\hline
\end{tabular}


Table 3/ Tabela 3. Comparison of the differences registered in different days $\left(\Delta_{1}=\mathrm{t}_{1}\right.$ $\left.\mathrm{t}_{0} ; \Delta_{2}=\mathrm{t}_{2}-\mathrm{t}_{0} ; \Delta_{3}=\mathrm{t}_{2}-\mathrm{t}_{1}\right)$ on control (C) and treated (T) hands, for all subjects

$$
(* \mathrm{p}<0,05)
$$

Comparação das diferenças registadas em diferentes momentos de medição $\left(\Delta_{1}=\mathrm{t}_{1}-\mathrm{t}_{0}\right.$; $\left.\Delta_{2}=\mathrm{t}_{2}-\mathrm{t}_{0} ; \Delta_{3}=\mathrm{t}_{2}-\mathrm{t}_{1}\right)$ nas mãos controlo (C) e tratada (T) de todos os voluntários

$$
(* \mathrm{p}<0,05)
$$

\begin{tabular}{lccc}
\hline \multirow{2}{*}{ Variable / Variável } & \multicolumn{3}{c}{ p value / valor p } \\
\cline { 2 - 4 } & $\boldsymbol{\Delta}_{\mathbf{1}} \mathbf{C}$ vs $\boldsymbol{\Delta}_{\mathbf{1}} \mathbf{T}$ & $\boldsymbol{\Delta}_{\mathbf{2}} \mathbf{C}$ vs $\boldsymbol{\Delta}_{\mathbf{2}} \mathbf{T}$ & $\boldsymbol{\Delta}_{\mathbf{3}} \mathrm{C}$ vs $\boldsymbol{\Delta}_{\mathbf{3}} \mathbf{T}$ \\
\hline $\begin{array}{c}\mathrm{TEWL} / \mathbf{P T E A} \\
\left(\mathrm{g} / \mathrm{h} / \mathrm{m}^{2}\right)\end{array}$ & 0.363 & $0.028^{*}$ & 0.208 \\
\hline
\end{tabular}

Table 4/ Tabela 4. Results (mean \pm standard deviation) obtained for the parameters $\mathrm{t}_{1 / 2 \text { evap }}$ and DWM $\quad(* \mathrm{p}<0.05)$

Resultados (média \pm desvio-padrão) obtidos para os parâmetros $\mathrm{t}_{1 / 2 \text { evap }} \mathrm{e}$ DWM $\quad(* \mathrm{p}<0,05)$

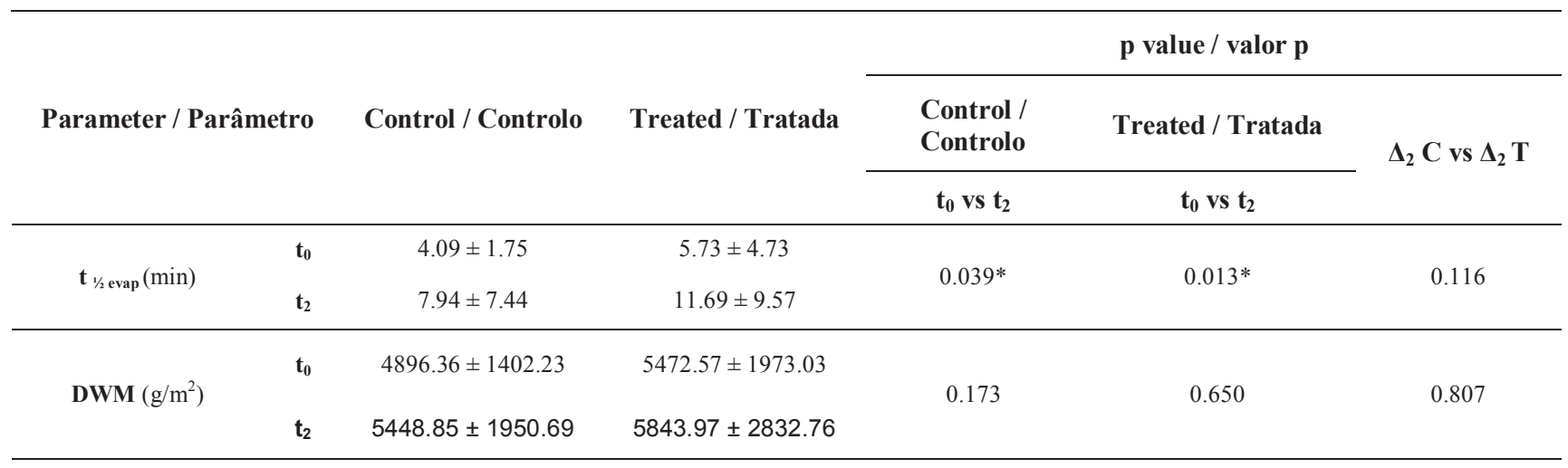

\section{Conclusions}

The model seems to be particularly sensitive to changes in skin water dynamics even when discrete, as in the present circumstances, and suggests that there is still room to improve these lateral effects of hand sanitizers on skin physiology.

\section{Conclusões}

Este modelo parece ser particularmente sensível a mudanças na dinâmica da água da pele, mesmo quando discretas como acontece nas actuais circunstâncias, e sugere a necessidade de continuar a melhorar estes efeitos secundários dos desinfectantes de mãos sobre a normal fisiologia da pele. 


\section{Acknowledgements}

The authors would like to express their thanks to all volunteers.

\section{Conflict of Interests}

The authors declare that there are no financial and/or personal relationships that could be viewed as presenting a potential conflict of interests.

\section{Agradecimentos}

Os autores expressam os seus agradecimentos a todos os voluntários.

\section{Conflito de Interesses}

Os autores declaram não existir qualquer relação pessoal ou financeira que possa ser entendida como representando um potencial conflito de interesses. 


\section{References / Referências}

1. Langley J. From soap and water, to waterless agents: Update on hand hygiene in health care settings. Can J Infect Dis 2002; 13(5):285-286.

2. Cowling BJ, Chan K, Fang VJ, Cheng CK, Fung RO, Wai W, et al. Facemasks and Hand Hygiene to Prevent Influenza Transmission in Households: A Cluster Randomized Trial. Ann Intern Med 2009; 151:437-446.

3. Larson EL, Early E, Cloonan P, Sugrue S, Parides M. An organizational climate intervention associated with increased handwashing and decreased nosocomial infections. Behav Med 2000; 26:14-22. 6.

4. Boyce JM, Pittet D; Healthcare Infection Control Practices Advisory Committee, HICPAC/SHEA/APIC/IDSA Hand Hygiene Task Force Guideline for hand hygiene in health-care settings. Recommendations of the Healthcare Infection Control Practices Advisory Committee and the HICPAC/ SHEA/APIC/IDSA Hand Hygiene Task Force. MMWR Recomm Rep 2002; 51(RR16): $1-45$.

5. Voss A, Widmer AF. No time for handwashing!? Handwashing versus alcoholic rub: can we afford $100 \%$ compliance? Infect Control Hosp Epidemiol 1997; 18:205-208.

6. Löffler H, Kampf G, Schmermund D, Maibach HI. How irritant is alcohol? Br J Dermatol 2007; 157(1):74-81.
7. Houben E, De Paepe K, Rogiers V. Skin condition associated with intensive use of alcoholic gels for hand disinfection: a combination of biophysical and sensorial data. Contact Dermatitis 2006; 54(5):261-7.

8. Lotte C, Rougier A, Wilson DR, Maibach HI. In vivo relationship between transepidermal water loss and percutaneous penetration of some organic compounds in man: effect of anatomic site. Arch Dermatol Res 1987; 279: 351-356.

9. van Neste H. In vivo evaluation of unbound water accumulation in stratum corneum. Dermatologica 1990: 181(3):197-201.

10. Tagami H, Kanamaru Y, Inoue K et al. Water sorption- desorption test of the skin in vivo for functional assessment of the stratum corneum. J Invest Dermatol 1982; 78(5):425428.

11. Berardesca E, Fideli D, Gabba P, Cespa M, Rabbiosi G, Maibach HI. Ranking of surfactant skin irritancy in vivo in man using the plastic occlusion stress test (POST). Contact Dermatitis 1990; 23 (1):1-5.

12. Rodrigues LM, Pinto PC, Galego N, Da Silva PA, Pereira LM. Transepidermal water loss kinetic modeling approach for the parameterization of skin water dynamics. Skin Res Technol 1999; 5:72-82.
13. Rosado C, Pinto P, Rodrigues LM. Modeling TEWL-desorption curves: a new practical approach for the quantitative in vivo assessment of skin barrier. Exp Dermatol 2005; 14(5):386-90.

14. Rosado C, Pinto P, Rodrigues LM. Assessment of moisturizers and barrier function restoration using dynamic methods. Skin Res Technol 2009; 15(1):77-83.

15. World Medical Association. World Medical Association Declaration of Helsinki: ethical principles for medical research involving human subjects. JAMA; 310(20):2191-4.

16. Panchagnula R, Salve PS, Thomas NS, Jain AK, Ramarao P. Transdermal delivery of naloxone: effect of water, propylene glycol, ethanol and their binary combinations on permeation through rat skin. Int J Pharm 2001; 219:95-105.

17. Manabe E, Sugibayashi K, Morimoto Y. Analysis of skin penetration enhancing effect of drugs by ethanol-water mixed systems with hydrodynamic pore theory. Int. J. Pharm. 1996; 129:211-221. 